Article

\title{
Baicalein Prevents 6-Hydroxydopamine-Induced Mitochondrial Dysfunction in SH-SY5Y Cells via Inhibition of Mitochondrial Oxidation and Up-Regulation of DJ-1 Protein Expression
}

\author{
Yue-Hua Wang ${ }^{1,2,3, *}$, Hai-Tao Yu ${ }^{4}$, Xiao-Ping Pu ${ }^{1, *}$ and Guan-Hua Du ${ }^{2,3, *}$ \\ 1 Department of Molecular and Cellular Pharmacology, School of Pharmaceutical Sciences, \\ Peking University; Beijing 100191, China \\ 2 Beijing Key Laboratory of Drug Target Identification, Institute of Materia Medica, \\ Chinese Academy of Medical Sciences \& Peking Union Medical College, Beijing 100050, China \\ 3 State Key Laboratory of Bioactive Substance and Function of Natural Medicines, \\ Institute of Materia Medica, Chinese Academy of Medical Sciences \& Peking Union Medical \\ College, Beijing 100050, China \\ 4 Jiangsu Kanon Pharmaceutical Co., Ltd, Lianyungang 222047, China \\ * Authors to whom correspondence should be addressed; E-Mails: wangyuehua@pku.org.cn (Y.-H.W.); \\ pxp123@bjmu.edu.cn (X.-P.P.); dugh@imm.ac.cn (G.-H.D.); Tel.: +86-10-6316-5313 (Y.-H.W.); \\ +86-10-8280-2431 (X.-P.P.); +86-10-6316-5184 (G.-H.D.).
}

Received: 24 September 2013; in revised form: 18 November 2013 / Accepted: 19 November 2013 / Published: 27 November 2013

\begin{abstract}
Parkinson's disease (PD) is a neurodegenerative disorder characterized by progressive loss of dopaminergic (DA) neurons at the substantia nigra. Mitochondrial dysfunction is involved in the mechanism of cell damage in Parkinson's disease (PD). 6-Hydroxydopamine (6-OHDA) is a dopamine analog which specifically damages dopaminergic neurons. Baicalein has been previously reported to have potential in the treatment of PD. The purpose of the present study was to investigate the mechanism of action of baicalein against 6-OHDA injury in SH-SY5Y cells. The results showed that baicalein significantly alleviated alterations of mitochondrial redox activity and mitochondrial membrane potential induced by 6-OHDA in a dose-dependent manner in SH-SY5Y cells compared with vehicle group. Futhermore, baicalein decreased the production of ROS and upregulated the DJ-1 protein expression in SH-SY5Y cells. In addition, baicalein also inhibited ROS production and lipid peroxidation $\left(\mathrm{IC}_{50}=6.32 \pm 0.03 \mu \mathrm{M}\right)$ in rat brain mitochondia. In summary, the underlying mechanisms of baicalein against
\end{abstract}


6-OHDA-induced mitochondrial dysfunction may involve inhibition of mitochondrial oxidation and upregulation of DJ-1 protein expression.

Keywords: baicalein; Parkinson's disease; 6-hydroxydopamine; SH-SY5Y cells; brain mitochondria

\section{Introduction}

Parkinson's disease (PD) is a neurodegenerative disorder characterized by slowly progressive degeneration of dopamine (DA) neurons in the substantia nigra pars compacta, with subsequent damage of nerve terminals, accompanied by DA depletion in the striatum. Although the neuropathological hallmarks of PD are well described, the etiology remains still undefined. Notably, several observations suggest that mitochondrial dysfunction and oxidative stress are involved in the pathogenesis of PD [1-3].

Many lines of evidence suggest that mitochondrial dysfunction plays a central role in the pathogenesis of PD [4]. Mitochondrion is an essential organelle that supplies the cell with ATP through oxidative phosphorylation, synthesizes key molecules, and buffers calcium gradients [5]. It is not surprising that mitochondrial health is tightly regulated and associated with the homeostasis and aging of the organism [6]. Mitochondrial diseases often have an associated metabolic component, and consequently mitochondrial defects are expected in aging, and other energy-dependent disturbances [7]. In such disturbances, cellular oxidative damage caused by the generation of reactive oxygen species (ROS) that exceed the natural antioxidant activity is likely an initiating factor in aging [8]. ROS cause oxidative damage to proteins, lipids, and DNA and are one of the most prominent factors related to neurodegeneration. Therefore, regulation of redox signaling and inhibiting excess ROS generation would contribute greatly not only to extend longevity but also to ameliorate the progression of dopaminergic cell death seen in patients with PD [9].

The protein DJ-1 was first identified as a novel oncogene product [10] and was later found to be a causative gene product of a familial form of PD (PARK7) [11]. DJ-1 plays important roles in transcriptional regulation [12], anti-oxidative stress reaction [13-16], and the elimination of abnormal protein aggregates [17]. DJ-1 is a ubiquitous redox-responsive cytoprotective protein with diverse functions. In addition to its direct quenching ROS effect within neurons, DJ-1 may play a role of neuroprotection in a transcellular manner in chronic neurodegenerative diseases [18].

6-Hydroxydopamine (6-OHDA) is a dopamine analog, which specifically damages dopaminergic neurons either via uncoupling mitochondrial oxidative phosphorylation resulting in energy deprivation or alternatively, is associated with its ability to produce hydrogen peroxide, hydroxyl and superoxide radicals under physiological $\mathrm{pH}$ conditions [19]. Evidence demonstrates that 6-OHDA generates ROS and induces apoptosis in dopaminergic cells of rat substantia nigra [20]. It has also been reported that 6-OHDA inhibits complexes I and IV of the mitochondrial respiratory chain [21,22]. Therefore, 6-OHDA is used to investigate the cellular and molecular mechanisms underlying selective degeneration of dopaminergic neurons in PD. The SH-SY5Y cell line has become a popular cell model for PD research because this cell line possesses many characateristics of DAergic neurons [23]. It has been used as an in vitro model for the study of PD and to determine the effect of protective and therapeutic agents. It is thought that 6-OHDA induces toxicity that mimics the neuropathological and biochemical characteristics of PD in 
SH-SY5Y cells [24-28]. Therefore, the 6-OHDA-induced SH-SY5Y cell toxicity was used as a vitro PD model in our studies to investigate the possible protective effect of baicalein.

Baicalein, a flavonoid obtained from the roots of the traditional Chinese herbal medicine Huangqin, Scutellaria baicalensis Georgi (Figure 1), has been widely used for treatment of inflammation, hypertension, cardiovascular disease, bacterial infection and cancer [29,30]. Our previous studies have shown that baicalein has anti-experimental Parkinsonism effects, especially against muscle tremors, in a mice model [31,32] and a rat model [33], however, the mechanisms and target protein(s) underlying this protective effect remain largely unknown. The purpose of this study was to explore the mechanism of action of baicalein against PD.

Figure 1. The chemical structure of baicalein.

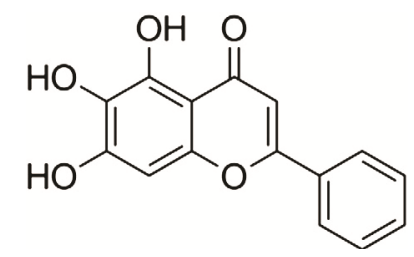

\section{Results and Discussion}

\subsection{Effect of Baicalein on Morphology and Cell Viability in SH-SY5Y Cells Damaged by 6-OHDA}

It is known that 6-OHDA could selectively cause degeneration of the nigrostriatal dopaminergic neuronal pathway in several animals [22] and cells [34,35], so 6-OHDA-damaged SH-SY5Y cells were used as an in vitro PD model in our studies to investigate the possible mechanism of action of baicalein. As shown in Figure 2A, within $24 \mathrm{~h}$ of treatment with 6-OHDA alone, the majority of SH-SY5Y cells had undergone morphological changes such as membrane blebbing and cell shrinkage. Co-treatment with baicalein protected the cells from 6-OHDA damage.

Figure 2. Effect of baicalein on the morphological changes and viability of SH-SY5Y cells induced by 6-OHDA. (A) Morphological changes were observed by light microscopy. Representative photographs showing control cells (a), vehicle cells (b), 6-OHDA + baicalein $0.1 \mu \mathrm{M}$ (c), 6-OHDA + baicalein $1 \mu \mathrm{M}$ (d), and 6-OHDA+baicalein $10 \mu \mathrm{M}$ (e). (B) Cell viability was estimated by MTT assay.

(A)

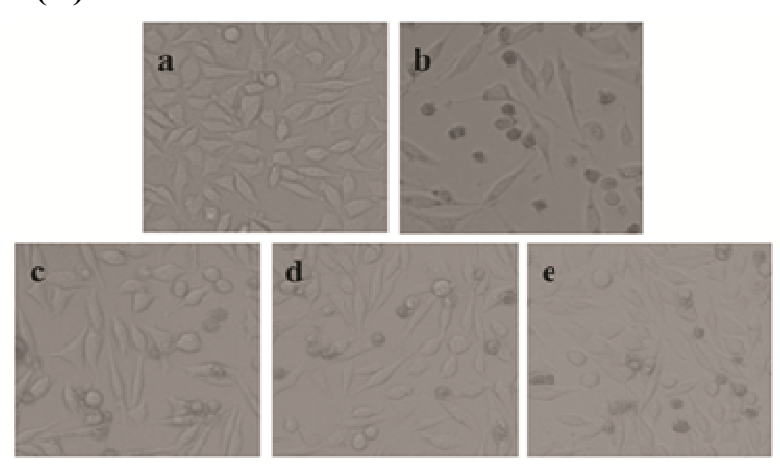

(B)

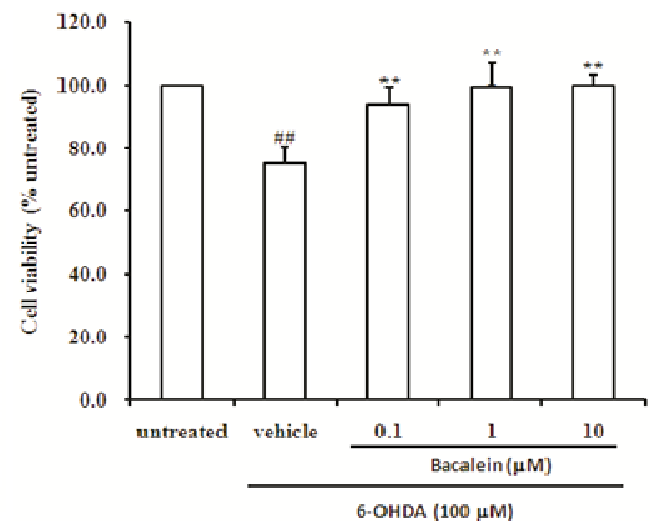

Data were expressed as percent cell viability of untreated cells. Data are the mean $\pm \mathrm{SD}, n=4,{ }^{\# \#} p<0.01 v s$. untreated group; $* * p<0.01 v s$. vehicle group. 
We also determined the cell viability by MTT assay, Treatment of SH-SY5Y cells with 6-OHDA alone resulted in an approximately $25 \%$ reduction in cell survival within $24 \mathrm{~h}$, whereas co-treatment with $0.1,1$ and $10 \mu \mathrm{M}$ baicalein all showed a reduction of 6-OHDA-mediated cytotoxicity (all $p<0.01$. Figure 2B). These results indicate that the incubation of SH-SY5Y cells with baicalein effectively prevents 6-OHDA-induced cytotoxicity.

\subsection{Baicalein Attenuates the Decrease of Mitochondria Redox Activity and the Collapse of Mitochondrial Membrane Potential Induced by 6-OHDA in SH-SY5Y Cells}

Mitochondrial dysfunction has long been implicated in the pathogenesis of Parkinson's disease (PD). The integrity of mitochondrial function is crucial for the maintenance of cell viability. Increasing evidence suggests that mitochondria are deeply involved in the production of reactive oxygen species through the electron carriers of the respiratory chain [36-39]. Mitochondrial dysfunction was detected as a decrease in mitochondrial redox activity and a loss in mitochondrial membrane potential ( $\Delta \psi m)$. Here, we used the resazurin staining method for the dectection mitochondrial redox activity and the JC-1 staining assay for the detection $\triangle \psi m$ in SH-SY5Y cells. Rezazurin is a fluorescent indicator of mitochondrial function. JC-1 is sensitive to mitochondrial membrane potential, and the changes in the ratio between aggregate (red) and monomer (green) fluorescence can provide information regarding the mitochondrial membrane potential. Thus, resazurin and JC-1 are valuable analytical tools for examining mitochondrial function [40]. The results showed that $24 \mathrm{~h}$ of incubation with 6-OHDA significantly reduced mitochondria redox activity compared to the untreated group $(p<0.01$, Figure $3 \mathrm{~A})$. On the other hand, co-treatment with $1 \mu \mathrm{M}$ and $10 \mu \mathrm{M}$ of baicalein significantly attenuated mitochondria redox activity impair induced by 6-OHDA (both $p<0.05$, Figure 3A). As shown in Figure 3B, treatment with $100 \mu \mathrm{M}$ of 6-OHDA for $24 \mathrm{~h}$ resulted in significant decrease of $\Delta \psi m$ compared with untreated group $(p<0.01$, Figure 3B).

Figure 3. Effect of baicalein on mitochondrial redox activity and mitochondria membrane potential of SH-SY5Y cells induced by 6-OHDA. (A) Mitochondrial redox activity was assayed by resazurin; (B) Mitochondria membrane potential was assayed by JC-1 staining. Data were expressed as percentage of untreated cells.
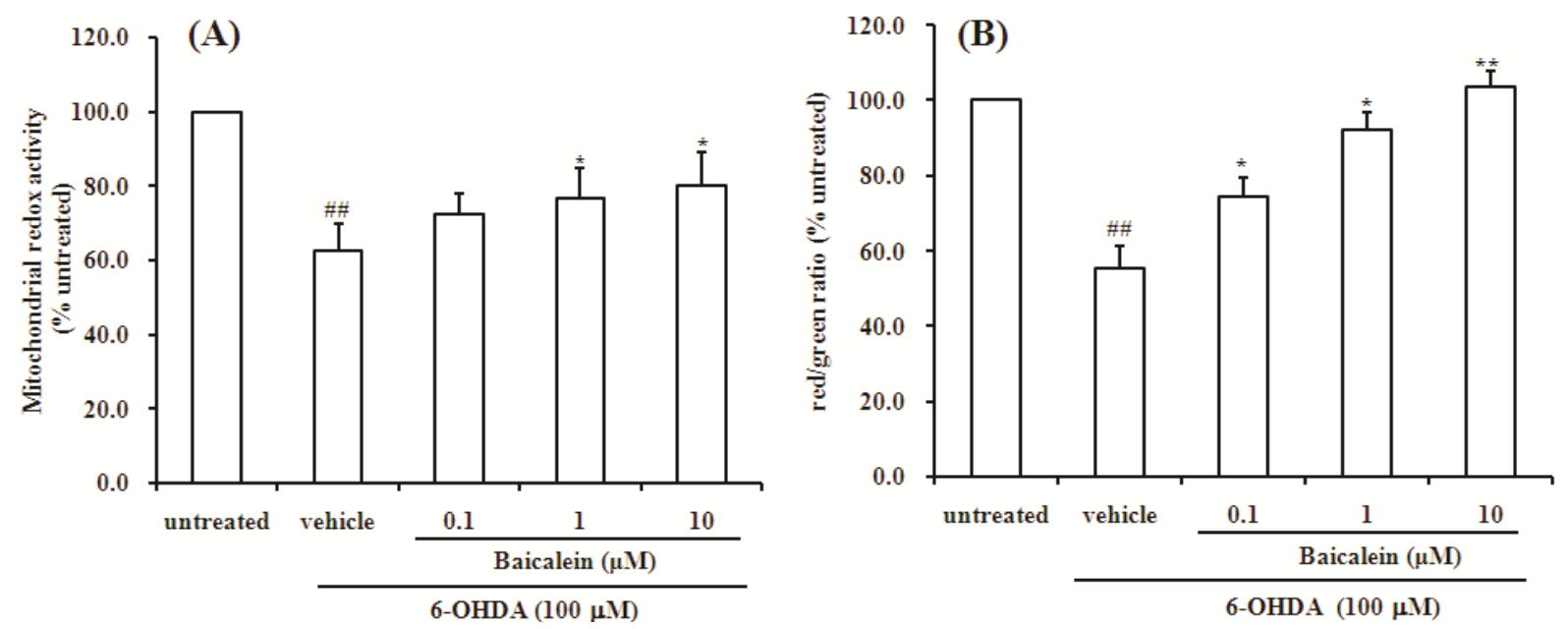

Data are the mean $\pm \mathrm{SD}, n=4,{ }^{\#} p<0.01$ vs. untreated group; ${ }^{*} p<0.05, * * p<0.01 v s$. vehicle group. 
However, the decrease of $\Delta \psi m$ induced by 6-OHDA was significantly attenuated by co-treatment with baicalein in a dose-dependent manner. The present study indicates that mitochondrial redox activity decrease and mitochondrial membrane potential dissipation may play important roles in 6-OHDA induced dopaminergic neurotoxicity, and further provides that improvement of mitochondrial dysfunction may be a better way to slow progressive dopaminergic neurodegeneration commonly associated with PD.

\subsection{Baicalein Reduces the Production of ROS Induced by 6-OHDA in SH-SY5Y Cells}

6-OHDA is a highly reactive substance, which is readily auto-oxidized and oxidatively deaminated by monoamine oxidase, to give rise to ROS [41], which in turn, can cause DNA strand breaks, damage protein residues and initiate lipid peroxidation reactions [42]. The intracellular ROS level was determined by DCF fluorescence. SH-SY5Y cells treated with 6-OHDA showed a significant increase (about 1.4-fold) of intracellular ROS compared with untreated cells ( $p<0.01$, Figure 4 ). This increase was significantly attenuated by co-incubation with $0.1,1$ and $10 \mu \mathrm{M}$ baicalein (all $p<0.01$, Figure 4). This result indicates that the co-incubation of SH-SY5Y cells with baicalein effectively prevents 6-OHDA-induced the production of ROS (Figure 4). These results indicate that ROS plays an important role in the induction of neuronal damage by 6-OHDA, and baicalein could inhibit the production of ROS in SH-SY5Y cells, which indicates that the blockage of ROS by baicalein might be a very important factor for its neuroprotection.

Figure 4. Effect of baicalein on generation of ROS induced by 6-OHDA in SH-SY5Y cells.

Data were expressed as percentage of untreated cells.

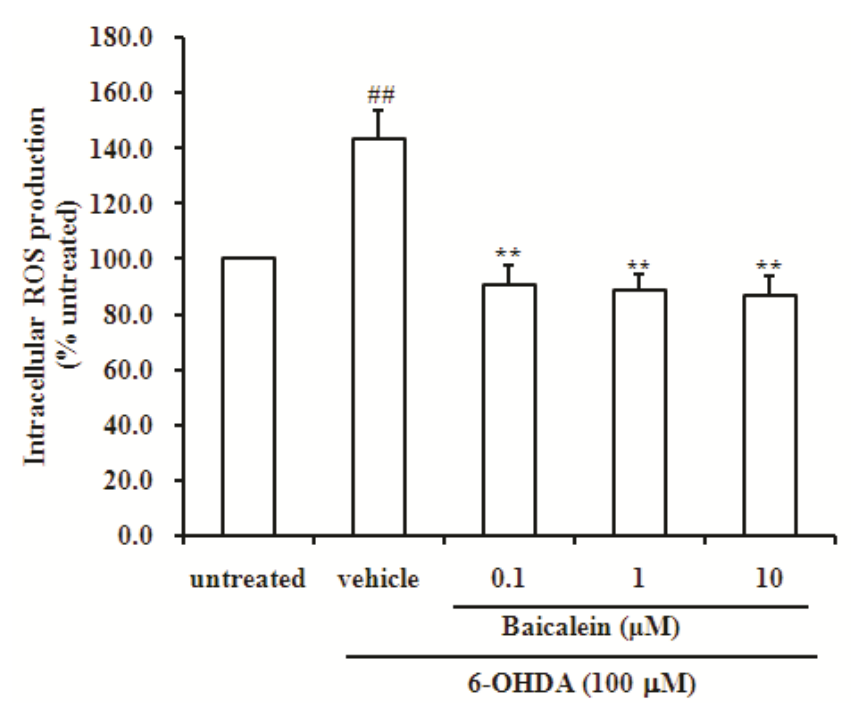

Data are the mean $\pm \mathrm{SD}, n=4, \# \# p 0.01 v s$. untreated group; ** $p<0.01 v s$. vehicle group.

\subsection{Baicalein Up-Regulates DJ-1 Protein Expression in SH-SY5Y Cells}

DJ-1 is known to play a key role in antioxidant and neuroprotection in neuronal cells $[13,43,44]$. DJ-1 regulates redox signaling kinase pathways and acts as a transcriptional regulator of antioxidative gene batteries. Therefore, DJ-1 is an important redox-reactive signaling intermediate controlling oxidative stress during age-related neurodegenerative processes. The anti-oxidant properties of DJ-1 lead to cytoprotection under oxidative stress conditions [45-47]. Thus, up-regulation of DJ-1 protein expression 
or augmenting DJ-1 activity might provide novel approaches to treating chronic neurodegenerative illnesses [18]. It is reported that DJ-1 binds to the mitochondrial complex I and plays a role in maintenance of mitochondrial complex I integrity [48].

As shown in Figure 5, treatment of SH-SY5Y cells with 6-OHDA alone for $24 \mathrm{~h}$ resulted in an approximately $22 \%$ reduction in DJ-1 protein $(p<0.01)$, whereas co-treatment with baicalein significantly increased DJ-1 protein expression in a dose-dependent manner in SH-SY5Y cells compared with vehicle group. This result suggests that baicalein effects reduction of cytotoxicity and mitochondrial dysfunction via up-regulation of DJ-1 protein. Our finding also indicates that DJ-1 is critical for mitochondrial function and may be the targeting protein for baicalein against PD.

Figure 5. Effect of baicalein on DJ-1 protein expression in SH-SY5Y cells induced by 6-OHDA. Data were expressed as percentage of untreated cells.

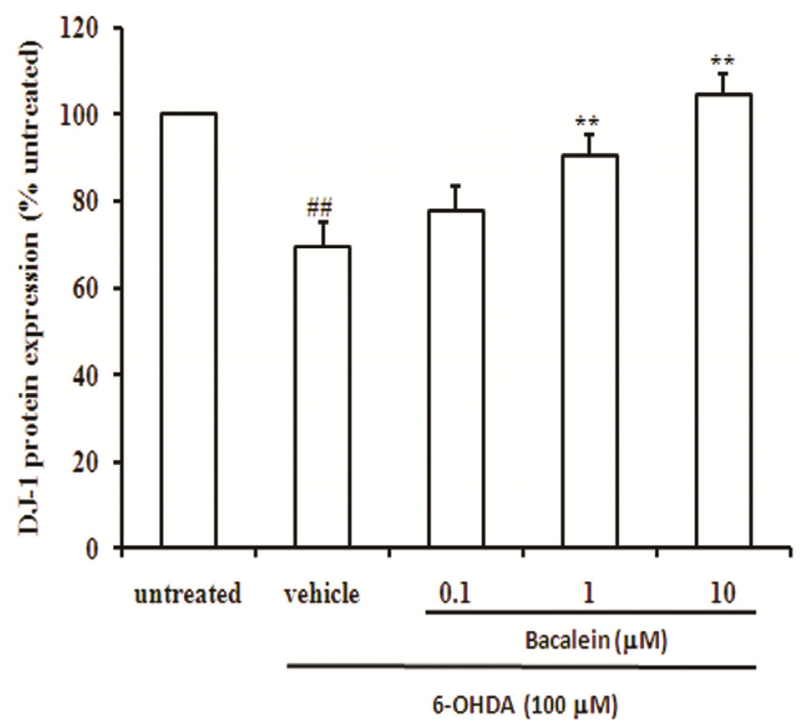

Data are the mean $\pm \mathrm{SD}, n=4, \# \# p<0.01 v s$. untreated group; $* * p<0.01 v s$. vehicle group.

\subsection{Baicalein Suppresses the ROS Production Induced by 6-OHDA and Lipid Peroxidation in Rat}

\section{Brain Mitochondria}

A wide variety of ROS are produced in the course of the normal metabolism in biological systems and they have several important physiological functions, but their accumulation beyond the required amount can potentially damage lipids, proteins, and nucleic acids [49].

To observe the direct effect of baicalein on mitochondrial oxidative stress, reactive oxidant species level was measured in isolated rat brain mitochondria in further research. Baicalein exhibited significant protective effects on 6-OHDA-induced reactive oxidant species production, which were in accordance with the results obtained from the cellular study (Figure 6A). In addition, baicalein also inhibited the lipid peroxidation of brain mitochondria in a concentration-dependent manner (Figure 6B). The $\mathrm{IC}_{50}$ value is $6.32 \pm 0.03 \mu \mathrm{M}$. Our results showed baicalein reduced the production of ROS in rat brain mitochondria and inhibit lipid peroxidation of rat brain mitochondria. 
Figure 6. Effect of baicalein on generation of ROS induced by 6-OHDA and lipid peroxidation in rat brain mitochondria. (A) ROS production in rat brain mitochondria; (B) lipid peroxidation in rat brain mitochondria.
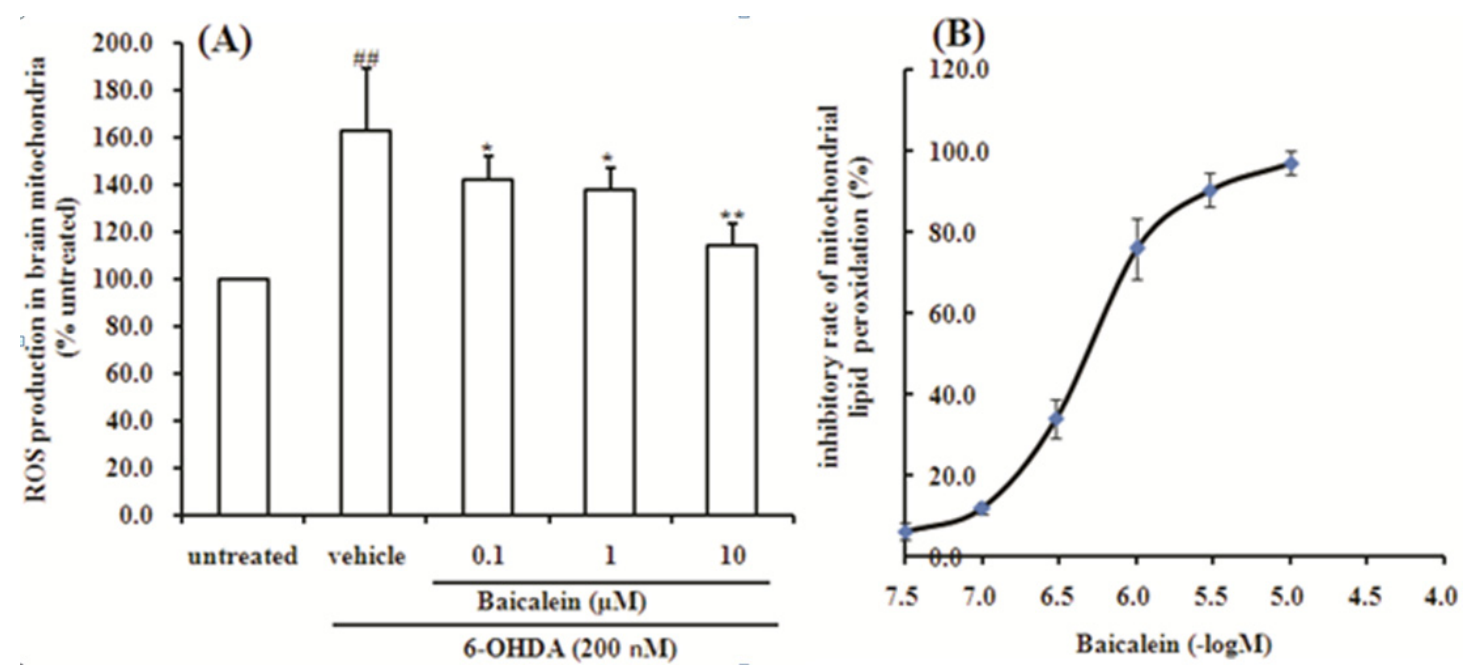

Data were expressed as percentage of untreated cells. Data are the mean $\pm \mathrm{SD}, n=4$, \#\#p $<0.01 v s$. untreated group; ** $p<0.01 v s$. vehicle group.

\section{Experimental}

\subsection{Materials}

Bacalein was obtained from National Institutes for Food and Drug Control (Beijing, China). The SH-SY5Y cell line was obtained from Cell Culture Centre, Institute of Basic Medical Sciences, Chinese Academy of Medical Science (Beijing, China). DMEM and fetal bovine serum were purchased from Gibco (Grand Island, NY, USA). 6-OHDA, MTT, 2,7-dichlorodihydrofluorescein diacetate $\left(\mathrm{H}_{2}\right.$ DCF-DA) and resazurin were purchased from Sigma-Aldrich (St. Louis, MO, USA). JC-1 assay kit was purchased from Beyotime Institute of Biotechnology (Haimen City, Jiangsu, China). DJ-1 Cell-Based ELISA Kit was a product of the Abnova Company (Taipei City, Taiwan).

\subsection{Cell Culture and Treatment}

SH-SY5Y cells were grown in DMEM supplemented with $10 \%$ fetal bovine serum at $37{ }^{\circ} \mathrm{C}$ in a humidified atmosphere containing 5\% $\mathrm{CO}_{2}$. Stock solution of 6-OHDA $(100 \mathrm{mM}, 1,000 \times)$ was dissolved in $0.2 \%$ ascorbic acid and aliquoted for storage at $-20{ }^{\circ} \mathrm{C}$. Before 6-OHDA was added to the culture medium, the stock solution was freshly diluted to the final concentration of $100 \mu \mathrm{M}$ with serum-free DMEM medium. The cells were plated into 96 -well plates at a density of $2 \times 10^{5}$ cells $/ \mathrm{mL}$. After $70 \%-80 \%$ confluence, the cells were pre-incubated with different concentration of baicalein in a serum-free DMEM medium for $1 \mathrm{~h}$. Then, 6-OHDA was added to the wells at a final concentration of $100 \mu \mathrm{M}$ and incubated for another $24 \mathrm{~h}$ at $37{ }^{\circ} \mathrm{C}$. In this study, the untreated group represented the control group which was treated with serum-free DMEM medium containing $0.0002 \%$ ascorbic acid, but without 6-OHDA. The vehicle group represented 6-OHDA-treated alone without baicalein. 


\subsection{Morphological Observations and Viability Assay}

Morphological changes were observed under microscope. Cell viability was evaluated by the MTT assay [50]. Briefly, after treatment, MTT $(0.5 \mathrm{mg} / \mathrm{mL})$ was added to each well and incubated for $4 \mathrm{~h}$ at $37^{\circ} \mathrm{C}$. Then, the supernatant was removed and the formazan product obtained was dissolved in $100 \mu \mathrm{L}$ dimethylsulfoxide (DMSO) with stirring for $15 \mathrm{~min}$ on a microtiter plate shaker and the absorbance was read at $540 \mathrm{~nm}$ using a Spectramax M5 microplate reader (Molecular Devices, Sunnyvale, CA, USA).

\subsection{Measurement of Mitochondrial Redox Activity by Resazurin}

Rezazurin is a fluorescent indicator of mitochondrial function. Upon oxidoreductase exposure in mitochondria, resazurin (blue and nonfluorescent) is reduced to resorufin (pink and highly florescent). The pink fluorescence intensity is examined at an excitation of $530 \mathrm{~nm}$ and an emission of $590 \mathrm{~nm}$. The change rate in fluorescence intensity is associated with mitochondrial redox activity. After treatment, resazurin at final concentration of $5 \mu \mathrm{M}$ were added into the wells and fluorescence intensity was examined at an excitation of $530 \mathrm{~nm}$ and an emission of $590 \mathrm{~nm}$. Then, the plate was incubated for another $60 \mathrm{~min}$ then fluorescence was measured. The changing rate was represented as $\left(\mathrm{F}_{60}-\mathrm{F}_{0}\right) / \mathrm{F}_{0} \times 100 \%$. $\mathrm{F}_{60}, \mathrm{~F}_{0}$ referred to fluorescence of $60 \mathrm{~min}$ and $0 \mathrm{~min}$ [51].

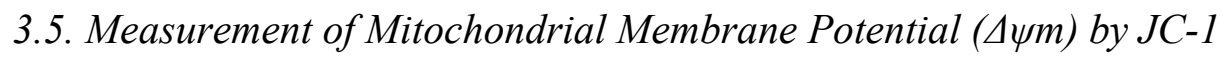

The fluorescent probe JC-1 (5,5',6,6'-tetrachloro-1,1',3,3'-tetraethylbenzimidazolylcarbocyanine iodide) was used to estimate of mitochondrial membrane potential $(\Delta \psi m)$ [52]. After treatment, the culture medium was removed and loaded with JC-1 solution for $15 \mathrm{~min}$ at $37^{\circ} \mathrm{C}$ in the dark. After two more rinses with Hank's solution, fluorescence intensity of the red/green ratio was determined at an excitation of $490 \mathrm{~nm}$ and emission of $530 \mathrm{~nm}$ (green fluorescent monomers) and $590 \mathrm{~nm}$ (red fluorescent aggregates) respectively.

\subsection{Determination of Intracellular ROS Level}

Intracellular ROS level was measured using 2,7-dichlorofluorescein-dictate $\left(\mathrm{H}_{2} \mathrm{DCF}-\mathrm{DA}\right)$ staining method [53]. After incubation with 6-OHDA, cells were loaded with $10 \mu \mathrm{M} \mathrm{H}_{2}$ DCFHDA for 30 min at $37^{\circ} \mathrm{C}$ in the dark. Cells were then analyzed on a Spectramax M5 microplate reader with excitation at $488 \mathrm{~nm}$ and emission at $525 \mathrm{~nm}$.

\subsection{DJ-1 Protein Expression Assay Using Cell-Based ELISA}

After treatment, the cells were quenched, fixed and blocked. Primary antibodies specific for DJ-1 protein and GAPDH were added and allowed to bind to their respective epitopes. Then, HRP-conjugated secondary antibodies were added and incubated for $1.5 \mathrm{~h}$ at room temperature with gentle shaking. After washing, TMB solution was added to each well and incubated for $30 \mathrm{~min}$ at room temperature, and then the stop solution was added to each well and read OD at $450 \mathrm{~nm}$ immediately using the microplate reader. After finishing reading the absorbance at $450 \mathrm{~nm}$, the plate was washed twice with Wash Buffer and twice with TBS and crystal violet was added to each well to bind with cell nuclei and give 
absorbance readings proportional to cell counts at $595 \mathrm{~nm}$. The measured $\mathrm{OD}_{450}$ readings can be normalized using the $\mathrm{OD}_{595}$ values via the proportion $\left(\mathrm{OD}_{450} / \mathrm{OD}_{595}\right)$.

\subsection{Isolation of Rat Brain Mitochondria}

Rat brain mitochondria were obtained by differential centrifugation [54]. Male Sprague-Dawley rats (250-300 g; Beijing Vital River Laboratory Animal Technology Co., Ltd; license: SCXK (JING) 2007-0001) were used in this study. All animal experiments were approved by the Laboratories Institutional Animal Care and Use Committee of Chinese Academy of Medical Sciences. Briefly, rats were decapitated and the brain was placed in beakers containing ice-cold isolation buffer $(0.25 \mathrm{M}$ sucrose containing $10 \mathrm{mM}$ Tris-HCl, $1 \mathrm{mM}$ EDTA, and $250 \mu \mathrm{g} / \mathrm{mL}$ BSA, pH 7.4). The brain tissue was weighed, repeatedly washed with the isolation buffer to remove adhering blood. Then, 10 volume (w/v) of homogenate was prepared in a glass homogenizer. The nuclei and cell debris were sedimented by centrifugation at $1,000 \mathrm{~g}$ for $10 \mathrm{~min}$ at $4{ }^{\circ} \mathrm{C}$ and discarded. The supernatant was collected for further centrifugation at $10,000 \mathrm{~g}$ for $10 \mathrm{~min}$ at $4{ }^{\circ} \mathrm{C}$. The sediment were washed, gently suspended in the isolation medium and centrifuged at $10,000 \mathrm{~g}$ for $10 \mathrm{~min}$ at $4{ }^{\circ} \mathrm{C}$ for further purification of the mitochondria. Finally, the sedimented mitochondria were suspended in the above buffer at a concentration of $10-15 \mathrm{mg} / \mathrm{mL}$. Mitochondrial protein concentration was determined by the Bradford method using BSA as a standard. Mitochondria were prepared fresh for each experiment and used within $4 \mathrm{~h}$ of isolation.

\subsection{Detection of ROS Production in Rat Brain Mitochondria}

ROS production in isolated rat brain mitochondria was monitored using the fluorescent probe $\mathrm{H}_{2}$ DCF-DA. After incubation for 30 min with $100 \mu \mathrm{M}$ 6-OHDA with or without baicalein pretreatment, mitochondria of different groups were incubated with $10 \mu \mathrm{M} \mathrm{H}_{2} \mathrm{DCF}-\mathrm{DA}$ at $37^{\circ} \mathrm{C}$ for another $30 \mathrm{~min}$, and the fluorescence intensity was measured at an excitation wavelength of $488 \mathrm{~nm}$ and emission wavelength of $525 \mathrm{~nm}$ in a microplate reader.

\subsection{Lipid Peroxidation of Rat Brain Mitochondria Induced by $\mathrm{FeSO}_{4}$-Cystine}

Lipid peroxidation was determined by the formation of thiobarbituric acid reactive substances (TBARS) as described and modification. Briefly, rat brain mitochondria $100 \mu \mathrm{g} / \mathrm{well}$ in $0.2 \mathrm{M}$ histidine buffer including $\mathrm{FeSO}_{4} 50 \mu \mathrm{M}$ and cystine $500 \mu \mathrm{M}$ was added into 96-well plate. Then, different concentration of baicalein were added and incubated at $37^{\circ} \mathrm{C}$ for $30 \mathrm{~min}$. The incubation was stopped by the addition of $1.0 \%$ thiobarbituric acid (TBA) solution and incubated for $30 \mathrm{~min}$ at $60{ }^{\circ} \mathrm{C}$. The absorption was measured at $532 \mathrm{~nm}$.

\subsection{Statistical Analysis}

The data were expressed as the means \pm SD. Significance of differences between group means was determined by One-way analysis of variance (ANOVA) followed by $t$-test. A $p$-value $<0.05$ was considered statistically significant. 


\section{Conclusions}

Here we have demonstrated that baicalein had neuroprotective effects against 6-OHDA-induced cytotoxicity and mitochondrial dysfunction in SH-SY5Y cells and brain mitochondria. The results indicate that baicalein protected against 6-OHDA-induced mitochondrial dysfunction by reducing intracellular ROS and up-regulation of DJ-1 protein expression. However, further studies should be conducted on the detailed mechanisms of how baicalein could reduce ROS and up-regulate DJ-1 protein expression.

\section{Acknowledgements}

This research was supported by grants from the National Natural Science Foundation of China (30973889), Science and Technology Major Projects: Significant New-Drugs Creation (2012ZX09103101-078; 2012ZX09103201-042; 2011ZX09401-014), the Research Special Fund for Public Welfare Industry of Health (200902008).

\section{Conflicts of Interest}

The authors declare no conflict of interest.

\section{References}

1. Kim, I.S.; Choi, D.K.; Jung, H.J. Neuroprotective effects of vanillyl alcohol in Gastrodia elata Blume through suppression of oxidative stress and anti-apoptotic activity in toxin-induced dopaminergic MN9D cells. Molecules 2011, 16, 5349-5361.

2. Swerdlow, R.H. The neurodegenerative mitochondriopathies. J. Alzheimers Dis. 2009, 17, 737-751.

3. Mosley, R.L.; Benner, E.J.; Kadiu, I.; Thomas, M.; Boska, M.D.; Hasan, K.; Laurie, C.; Gendelman, H.E. Neuroinflammation, oxidative stress and the pathogenesis of Parkinson's disease. Clin. Neurosci. Res. 2006, 6, 261-281.

4. Winklhofer, K.F.; Haass, C. Mitochondrial dysfunction in Parkinson's disease. Biochim. Biophys. Acta 2010, 1802, 29-44.

5. Frazier, A.E.; Kiu, C.; Stojanovski, D.; Hoogenraad, N.J.; Ryan, M.T. Mitochondrial morphology and distribution in mammalian cells. Biol. Chem. 2006, 387, 1551-1558.

6. Westermann, B. Bioenergetic role of mitochondrial fusion and fission. Biochim Biophys. Acta 2012, 1817, 1833-1838.

7. Chan, D.C. Mitochondria: Dynamic organelles in disease, aging, and development. Cell 2006, 125, 1241-1252.

8. Dröge, W.; Schipper, H.M. Oxidative stress and aberrant signaling in aging and cognitive decline. Aging Cell 2007, 6, 361-370.

9. Koppula, S.; Kumar, H.; More, S.V.; Lim, H.W.; Hong, S.M.; Choi, D.K. Recent updates in redox regulation and free radical scavenging effects by herbal products in experimental models of Parkinson's disease. Molecules 2012, 17, 11391-11420.

10. Nagakubo, D.; Taira, T.; Kitaura, H.; Ikeda, M.; Tamai, K.; Iguchi-Ariga, S.M.; Ariga, H. DJ-1, a novel oncogene which transforms mouse NIH3T3 cells in cooperation with ras. Biochem. Biophys. Res. Commun. 1997, 231, 509-513. 
11. Bonifati, V.; Rizzu. P.; van Baren, M.J.; Schaap, O.; Breedveld, G.J.; Krieger, E.; Dekker, M.C.; Squitieri, F.; Ibanez, P.; Joosse, M.; et al. Mutations in the DJ-1 gene associated with autosomal recessive early-onset Parkinsonism. Science 2003, 299, 256-259.

12. Clements, C.M.; McNally, R.S.; Conti, B.J.; Mak, T.W.; Ting, J.P. DJ-1, a cancer- and Parkinson's disease-associated protein, stabilizes the antioxidant transcriptional master regulator Nrf2. Proc. Nat. Acad. Sci. USA 2006, 103, 15091-15096.

13. Taira, T.; Saito, Y.; Niki, T.; Iguchi-Ariga, S.M.; Takahashi, K.; Ariga, H. DJ-1has a role in antioxidative stress to prevent cell death. EMBO Rep. 2004, 5, 213-218.

14. Yokota, T.; Sugawara, K.; Ito, K.; Takahashi, R.; Ariga, H.; Mizusawa, H. Down regulation of DJ-1 enhances cell death by oxidative stress, ER stress, and proteasome inhibition. Biochem. Biophys. Res. Commun. 2003, 312, 1342-1348.

15. Canet-Avilés, R.M.; Wilson, M.A.; Miller, D.W.; Ahmad, R.; McLendon, C.; Bandyopadhyay, S.; Baptista, M.J.; Ringe, D.; Petsko, G.A.; Cookson, M.R. The Parkinson's disease protein DJ-1 is neuroprotective due to cysteine-sulfonic acid-driven mitochondrial localization. Proc. Natl. Acad. Sci. USA 2004, 101, 9103-9108.

16. Martinat, C.; Shendelman, S.; Jonason, A.; Leete, T.; Beal, M.F.; Yang, L.; Floss, T.; Abeliovich, A. Sensitivity to oxidative stress in DJ-1-deficient dopamine neurons: An ES-derived cell model of primary Parkinsonism. PLoS Biol. 2004, 2, e327.

17. Bonifati, V.; Rizzu, P.; Squitieri, F.; Krieger, E.; Vanacore, N.; van Swieten, J.C.; Brice, A.; van Duijn, C.M.; Oostra, B.; Meco, G.; et al. DJ-1( PARK7), a novel gene for autosomal recessive, early onset parkinsonism. Neurol. Sci. 2003, 24, 159-160.

18. Kahle, P.J.; Waak, J.; Gasser, T. DJ-1 and prevention of oxidative stress in Parkinson's disease and other age-related disorders. Free Radic. Biol. Med. 2009, 47, 1354-1361.

19. Soto-Otero, R.; Méndez-Alvarez, E.; Sánchez-Iglesias, S.; Zubkov, F.I.; Voskressensky, L.G.; Varlamov, A.V.; de Candia, M.; Altomare, C. Inhibition of 6-hydroxydopamine-induced oxidative damage by 4,5-dihydro-3H-2-benzazepine $N$-oxides. Biochem. Pharmacol. 2008, 75, 1526-1537.

20. Soto-Otero, R.; Méndez-Alvarez, E.; Hermida-Ameijeiras, A.; López-Real, A.M.; Labandeira-García, J.L. Effects of (-)-nicotine and (-)-cotinine on 6-hydroxydopamine-induced oxidative stress and neurotoxicity: Relevance for Parkinson's disease. Biochem. Pharmacol. 2002, 64, 125-135.

21. Glinka, Y.Y.; Tipton, K.F.; Youdim, M.B. Nature of inhibition of mitochondrial respiratory complex I by 6-Hydroxydopamine. J. Neurochem. 1996, 66, 2004-2010.

22. Glinka, Y.Y.; Youdim, M.B. Inhibition of mitochondrial complexes I and IV by 6-hydroxydopamine. Eur. J. Pharmacol. 1995, 292, 329-332.

23. Xie, H.R.; Hu L.S.; Li G.Y. SH-SY5Y human neuroblastoma cell line: In vitro cell model of dopaminergic neurons in Parkinson's disease. Chin. Med. J. 2010, 123, 1086-1092.

24. Elkon, H.; Melamed, E.; Offen, D. 6-Hydroxydopamine increases ubiquitin-conjugates and protein degradation: Implications for the pathogenesis of Parkinson's disease. Cell Mol. Neurobiol. 2001, 21, 771-781.

25. Jordán, J.; Galindo, M.F.; Tornero, D.; González-García, C.; Ceña, V. Bcl-x L blocks mitochondrial multiple conductance channel activation and inhibits 6-OHDA-induced death in SH-SY5Y cells. J. Neurochem. 2004, 89, 124-133. 
26. Lin, Y.C.; Uang, H.W.; Lin, R.J.; Chen, I.J.; Lo, Y.C. Neuroprotective effects of glyceryl nonivamide against microglia-like cells and 6-hydroxydopamine-induced neurotoxicity in SH-SY5Y human dopaminergic neuroblastoma cells. J. Pharmacol. Exp. Ther. 2007, 323, 877-887.

27. Gomez-Lazaro, M.; Bonekamp, N.A.; Galindo, M.F.; Jordán, J.; Schrader, M. 6-Hydroxydopamine (6-OHDA) induces Drp1-dependent mitochondrial fragmentation in SH-SY5Y cells. Free Radic. Biol. Med. 2008, 44, 1960-1969.

28. Xiang, B.; Fei, X.; Zhuang, W.; Fang, Y.; Qin, Z.; Liang, Z. Cathepsin L is involved in 6-hydroxydopamine induced apoptosis of SH-SY5Y neuroblastoma cells. Brain Res. 2011, 1387, 29-38.

29. Ciesielska, E.; Gwardys, A.; Metodiewa, D. Anticancer, antiradical and antioxidative actions of novel Antoksyd S and its major components, baicalin and baicalein. Anticancer Res. 2002, 22, 2885-2891.

30. Li-Weber, M. New therapeutic aspects of flavones: The anticancer properties of Scutellaria and its main active constituents Wogonin, Baicalein and Baicalin. Cancer Treat. Rev. 2009, 35, 57-68.

31. Cheng, Y.; He, G.; Mu, X.; Zhang, T.; Li, X.; Hu, J.; Xu, B.; Du, G. Neuroprotective effect of baicalein against MPTP neurotoxicity: Behavioral, biochemical and immunohistochemical profile. Neurosci. Lett. 2008, 441, 16-20.

32. Mu, X.; He, G.R.; Yuan, X.; Li, X.X.; Du, G.H. Baicalein protects the brain against neuron impairments induced by MPTP in C57BL/6 mice. Pharmacol. Biochem. Behav. 2011, 98, 286-291.

33. Mu, X.; He, G.; Cheng, Y.; Li, X.; Xu, B.; Du, G. Baicalein exerts neuroprotective effects in 6-hydroxydopamine-induced experimental parkinsonism in vivo and in vitro. Pharmacol. Biochem. Behav. 2009, 92, 642-648.

34. Xu, R.; Liu, J.; Chen, X.; Xu, F.; Xie, Q.; Yu, H.; Guo, Q.; Zhou, X.; Jin, Y. Ribozyme-mediated inhibition of caspase-3 activity reduces apoptosis induced by 6-hydroxydopamine in PC12 cells. Brain Res. 2001, 899, 10-19.

35. Jin, C.F.; Shen, S.R.; Zhao, B.L. Different effects of five catechins on 6-hydroxydopamine-induced apoptosis in PC12 cells. J. Agric. Food Chem. 2001, 49, 6033-6038.

36. Beal, M.F. Parkinson's disease: A model dilemma. Nature 2010, 466, S8-S10.

37. Murphy, M.P. How mitochondria produce reactive oxygen species. Biochem. J. 2009, 417, 1-13.

38. Starkov, A.A. The role of mitochondria in reactive oxygen species metabolism and signaling. Ann. N. Y. Acad. Sci. 2008, 1147, 37-52.

39. Lambert, A.J.; Brand, M.D. Reactive oxygen species production by mitochondria. Methods Mol. Biol. 2009, 554, 165-181.

40. Nakai, M; Mori, A.; Watanabe, A.; Mitsumoto, Y. 1-methyl-4-phenylpyridinium (MPP+) decreases mitochondrial oxidation-reduction (REDOX) activity and membrane potential (Deltapsi(m)) in rat striatum. Exp. Neurol. 2003, 179, 103-110.

41. Levites, Y.; Youdim, M.B.; Maor, G.; Mandel, S. Attenuation of 6-hydroxydopamine (6-OHDA)-induced nuclear factor-kappaB (NF-kappaB) activation and cell death by tea extracts in neuronal cultures. Biochem. Pharmacol. 2002, 63, 21-29.

42. Halliwell, B. Reactive oxygen species, and the central nervous system. J. Neurochem. 1992, 59, 1609-1623. 
43. Yanagida, T.; Takata, K.; Inden, M.; Kitamura, Y.; Taniguchi, T.; Yoshimoto, K.; Taira, T.; Ariga, H. Distribution of DJ-1, Parkinson's diseaserelated protein PARK7, and its alteration in 6-hydroxydopamine-treated hemiparkinsonian rat brain. J. Pharmacol. Sci. 2006, 102, 243-247.

44. Yanagisawa, D.; Kitamura, Y.; Inden, M.; Takata, K.; Taniguchi, T.; Morikawa, S.; Morita, M.; Inubushi, T.; Tooyama, I.; Taira, T.; et al. DJ-1 protects against neurodegeneration caused by focal cerebral ischemia and reperfusion in rats. J. Cereb. Blood Flow Metab. 2008, 28, 563-578.

45. Lev, N.; Ickowicz, D.; Melamed, E.; Offen, D. Oxidative insults induce DJ-1 upregulation and redistribution: Implications for neuroprotection. Neurotoxicology 2008, 29, 397-405.

46. Yanagida, T.; Kitamura, Y.; Yamane, K.; Takahashi, K.; Takata, K.; Yanagisawa, D.; Yasui, H.; Taniguchi, T.; Taira, T.; Honda, T.; et al. Protection against oxidative stress-induced neurodegeneration by a modulator for DJ-1, the wild-type of familial Parkinson's disease-linked PARK7. J. Pharmacol. Sci. 2009, 109, 463-468.

47. Yanagida, T.; Tsushima, J.; Kitamura, Y.; Yanagisawa, D.; Takata, K.; Shibaike, T.; Yamamoto, A.; Taniguchi, T.; Yasui, H.; Taira, T.; et al. Oxidative stress induction of DJ-1 protein in reactive astrocytes scavenges free radicals and reduces cell injury. Oxid. Med. Cell Longev. 2009, 2, 36-42.

48. Hayashi, T.; Ishimori, C.; Takahashi-Niki, K.; Taira, T.; Kim, Y.C.; Maita, H.; Maita, C.; Ariga, H.; Iguchi-Ariga, S.M. DJ-1 binds to mitochondrial complex I and maintains its activity. Biochem. Biophys. Res. Commun. 2009, 390, 667-672.

49. Schapira, A.H. Mitochondrial dysfunction in neurodegenerative disorders. Biochim. Biophys. Acta 1998, 1366, 225-233.

50. Wang, Y.H.; Du, G.H. Ginsenoside Rg1 inhibits beta-secretase activity in vitro and protects against Abeta-induced cytotoxicity in PC12 cells. J. Asian Nat. Prod. Res. 2009, 11, 604-612.

51. Magnani, E.; Bettini, E. Resazurin detection of energy metabolism changes in serum-starved PC12 cells and of neuroprotective agent effect. Brain Res. Protoc. 2000, 5, 266-272.

52. Mashimo, K.; Ohno, Y. Ethanol hyperpolarizes mitochondrial membrane potential and increases mitochondrial fraction in cultured mouse myocardial cells. Arch. Toxicol. 2006, 80, 421-428.

53. Hwang, D.S.; Kim, H.G.; Kwon, H.J.; Cho, J.H.; Lee, C.H.; Lee, J.M.; Jang, J.B.; Kim, Y.S.; Lee, K.S.; Oh, M.S. Dangguijakyak-san, a medicinal herbal formula, protects dopaminergic neurons from 6-hydroxydopamine-induced neurotoxicity. J. Ethnopharmacol. 2011, 133, 934-939.

54. Wang, Y.H.; Xuan, Z.H.; Tian, S.; He, G.R.; Du, G.H. Myricitrin attenuates 6-hydroxydopamine-induced mitochondrial damage and apoptosis in PC12 cells via inhibition of mitochondrial oxidation. J. Func. Foods 2013, 5, 337-345.

Sample Availability: Samples of the compounds are available from the authors.

(C) 2013 by the authors; licensee MDPI, Basel, Switzerland. This article is an open access article distributed under the terms and conditions of the Creative Commons Attribution license (http://creativecommons.org/licenses/by/3.0/). 